\title{
NEUMANN SERIES OF BESSEL FUNCTIONS. II \\ BY
}

J. ERNEST WILKINS, JR.

1. Introduction. The Neumann series representation of a function $f(x)$ defined when $x \geqq 0$ is $\left[3\right.$, p. 359] $\left({ }^{1}\right)$

$$
s_{\nu}(x)=\sum_{n=0}^{\infty} a_{n v} J_{\nu+2 n+1}(x),
$$

in which $a_{n v}$ is defined for real values of $\nu$ as

$$
a_{n \nu}=2(\nu+2 n+1) \int_{0}^{\rightarrow \infty} t^{-1} f(t) J_{\nu+2 n+1}(t) d t .
$$

Let $A_{\nu}$ be the class of functions $f(t)$ such that $t^{\nu} f(t) /(1+t)^{p+\nu+1 / 2}$ is in $L(0, \infty)$ for some non-negative integer $p$ and such that the limits

$$
\begin{aligned}
& \int_{a}^{\rightarrow \infty} t^{-3 / 2} f(t) \cos \left(t-\frac{\nu \pi}{2}+\frac{\pi}{4}\right) d t, \\
& \int_{a}^{\rightarrow \infty} t^{-5 / 2} f(t) \sin \left(t-\frac{\nu \pi}{2}+\frac{\pi}{4}\right) d t,
\end{aligned}
$$

exist when $a>0$. The author [3] has proved the following theorems.

THEOREM 1.1. If $f(t)$ belongs to $A_{\nu}$, then the series

$$
x^{-\nu} S_{\nu}(x)=\sum_{n=0}^{\infty} 2(\nu+2 n+1) x^{-\nu} J_{\nu+2 n+1}(x) \int_{0}^{\rightarrow \infty} t^{-1} f(t) J_{\nu+2 n+1}(t) d t
$$

defines an entire function $x^{-\nu} s_{\nu}(x)$.

Theorem 1.2. Suppose that $\nu=0$ or -1 and $x>0$. If $f(t)$ belongs to $\mathcal{A}_{\nu}$ and is absolutely continuous on $(0, x)$, then we have that

$$
s_{\nu}(x)=f(x-)-f(0+) J_{0}(x)-\int_{0}^{x} J_{0}(x-v) F(v) d v,
$$

where $f(t)$ is defined for negative values of $t$ as $(-1)^{\nu-1} f(-t)$ and

$$
F(t)=f^{\prime}(t)-\frac{1}{2} \int_{0}^{\infty} v^{-1} J_{1}(v)\{f(t+v)-f(t-v)\} d v .
$$

Presented to the Society, February 25, 1950; received by the editors August 13, 1949.

(1) Numbers in brackets refer to the bibliography at the end of the paper. 
THEOREM 1.3. Suppose that $x>0$ and that either $\nu>-1$ or $\nu$ is an integer. Then if $f(t)$ belongs to $A_{\nu}$ and is of bounded variation in some neighborhood of $\dot{x}$, we have that

(1.8) $s_{\nu}(x)=\frac{1}{2}\{f(x+)+f(x-)\}-x \lim _{N=\infty, a=0} \int_{1}^{\rightarrow \infty} r J_{\nu}(x r) d r \int_{a}^{N} f(t) J_{\nu}(t r) d t$.

We propose in this paper to show that these three theorems remain true when the class $\mathcal{A}_{\nu}$ is replaced by the larger class $\mathcal{A}_{\nu}^{*}$ of functions $f(t)$ such that $t^{\nu} f(t)$ is in $L(0, a)$ for each positive $a$ and the limits (1.3) and (1.4) exist, or by the class $B_{\nu}$ of functions $f(t)$ such that $t^{\nu} f(t)$ is in $L(0, a)$ for each positive $a$ and the limits

$$
\begin{aligned}
& \int_{a}^{\rightarrow \infty} t^{-1} f(t) J_{\nu+1}(t) d t, \\
& \int_{a}^{\rightarrow \infty} t^{-2} f(t) J_{\nu}(t) d t
\end{aligned}
$$

exist when $a>0$. We first show in $\S 2$ that $A_{\nu}^{*}=\mathscr{B}_{\nu}$. In $\S 3$ we show that our results are best possible in the sense that if $f(t)$ is not in $\mathcal{B}_{\nu}$, then the coeffcients $a_{n \nu}$ defined by equation (1.2) will not all exist, so that if the question of convergence of the series (1.1) is to be meaningful, $f(t)$ must be in $\mathcal{B}_{\nu}$. In $\S \S 4$ and 5 we prove the indicated generalizations of Theorems $1.1,1.2$, and 1.3. In $\$ 6$ we show that the present theory is an actual extension of that in [3] by exhibiting a function $f(t)$ in $A_{\nu}^{*}$ which is not in $A_{\nu}$.

2. The proof that the classes $\mathcal{A}_{\nu}^{*}$ and $\mathcal{B}_{\nu}$ are identical. Let the functions $P(t, \nu)$ and $Q(t, \nu)$ be defined by the equations

$$
\begin{aligned}
& J_{\nu}(t)=(2 / \pi t)^{1 / 2}\left[P(t, \nu) \sin \left(t-\delta_{\nu}\right)+Q(t, \nu) \cos \left(t-\delta_{\nu}\right)\right], \\
& Y_{\nu}(t)=(2 / \pi t)^{1 / 2}\left[Q(t, \nu) \sin \left(t-\delta_{\nu}\right)-P(t, \nu) \cos \left(t-\delta_{\nu}\right)\right],
\end{aligned}
$$

where $\delta_{\nu}=\nu \pi / 2-\pi / 4$. Then it is known [2, p. 199] that $P(t, \nu)$ and $Q(t, \nu)$ admit the following asymptotic expansions:

$$
\begin{aligned}
& P(t, \nu) \sim \sum_{m=0}^{\infty}(-1)^{m}(\nu, 2 m)(2 t)^{-2 m} \\
& Q(t, \nu) \sim \sum_{m=0}^{\infty}(-1)^{m}(\nu, 2 m+1)(2 t)^{-2 m-1}
\end{aligned}
$$

in which $(\nu, q)=\Gamma(\nu+q+1 / 2) / q ! \Gamma(\nu-q+1 / 2)$. The following lemma may be proved readily with the help of the well known recurrence relations $[2, \mathrm{pp}$. $45,66]$

$$
J_{\nu-1}-J_{\nu+1}=2 J_{\nu}^{\prime}, \quad Y_{\nu-1}-Y_{\nu+1}=2 Y_{\nu}^{\prime}
$$


satisfied by the Bessel functions.

Lemma 2.1. The asymptotic expressions for $P(t, \nu)$ and $Q(t, \nu)$ may be differentiated term by term.

To prove that $A_{\nu}^{*}=B_{\nu}$ we shall also need the following lemma, whose proof can be made easily with the help of the second theorem of the mean for integrals.

LEMMA 2.2. Let $h(t)$ be in $L(a, b)$ for every $a$ and $b$ such that $Z \leqq a<b$, and suppose that the limit

$$
\int_{z}^{\rightarrow \infty} h(t) d t
$$

exists. If $g(t)$ is of bounded variation on $(Z, \infty)$, in particular if $g(t)$ is absolutely continuous on every finite interval $(Z, b)$ and

$$
\int_{z}^{\infty}\left|g^{\prime}(t)\right| d t<+\infty
$$

then the limit

$$
\int_{z}^{\rightarrow \infty} h(t) g(t) d t
$$

also exists.

Suppose that $f(t)$ is in $\mathcal{A}_{\nu}^{*}$. If we use equation (2.1) and Lemma 2.2 with $h(t)=t^{-3 / 2} f(t) \cos \left(t-\delta_{\nu}\right), g(t)=P(t, \nu+1)$, and $t^{-1} Q(t, \nu)$, and $h(t)=t^{-5 / 2} f(t)$ sin $\left(t-\delta_{\nu}\right), g(t)=t Q(t, \nu+1)$, and $P(t, \nu)$, we infer that the limits (1.9) and (1.10) exist and hence that $A_{\nu}^{*} \subset \mathcal{B}_{\nu}$. The fact that the inequality (2.3) holds with these choices of $g(t)$ follows from Lemma 2.1.

To prove the opposite inclusion we solve equation (2.1) and

$$
J_{\nu+1}(t)=(2 / \pi t)^{1 / 2}\left[-P(t, \nu+1) \cos \left(t-\delta_{\nu}\right)+Q(t, \nu+1) \sin \left(t-\delta_{\nu}\right)\right],
$$

and find that

$$
\begin{aligned}
& \cos \left(t-\delta_{\nu}\right)=J_{\nu}(t) P_{\nu}(t)+J_{\nu+1}(t) Q_{\nu}(t), \\
& \sin \left(t-\delta_{\nu}\right)=J_{\nu}(t) R_{\nu}(t)+J_{\nu+1}(t) S_{\nu}(t),
\end{aligned}
$$

in which

$$
\begin{array}{rlrl}
P_{\nu}(t) & =Q(t, \nu+1) T(t, \nu), & & Q_{\nu}(t)=-P(t, \nu) T(t, \nu) \\
R_{\nu}(t) & =P(t, \nu+1) T(t, \nu), & & S_{\nu}(t)=Q(t, \nu) T(t, \nu) \\
T(t, \nu) & =(\pi t / 2)^{1 / 2}[P(t, \nu) P(t, \nu+1)+Q(t, \nu) Q(t, \nu+1)]^{-1} .
\end{array}
$$

If $f(t)$ is in $\mathcal{B}_{\nu}$, we see from Lemma 2.2 that $f(t)$ will be in $\mathcal{A}_{\nu}^{*}$ provided the 
functions $t^{-1 / 2} Q_{\nu}(t), t^{1 / 2} P_{\nu}(t), t^{-1 / 2} R_{\nu}(t)$ and $t^{-3 / 2} S_{\nu}(t)$ satisfy the inequality (2.3). This statement is an immediate consequence of Lemma 2.1 and the definitions of these functions.

Thus we have proved that $A_{\nu}^{*}=\mathscr{B}_{\nu}$.

3. Existence of the coefficients $a_{n v}$. We shall prove the following theorem.

ThEOREM 3.1. The quantities $a_{n \nu}$ defined in equation (1.2) all exist if and only if $f(t)$ is in $\mathcal{B}_{\nu}$.

Let the Lommel polynomial $R_{m \mu}(t)$ be defined as $[2$, p. 296]

$$
R_{m \mu}(t)=\sum_{k=0}^{[m / 2]}(-1)^{k}(t / 2)^{2 k-m} \Gamma(\mu+m-k)(m-k) ! / k ! \Gamma(\mu+k)(m-2 k) !,
$$

where $[m / 2]$ is the largest integer which does not exceed $m / 2$. These polynomials have the property that $[2$, p. 294]

$$
J_{\nu+2 n+1}(t)=R_{2 n, v+1}(t) J_{\nu+1}(t)-R_{2 n-1, v+2}(t) J_{\nu}(t) .
$$

Let us suppose temporarily that $\nu>-1$ and define

$$
\begin{aligned}
& b_{n \nu}(N)=2(\nu+2 n+1) \int_{N}^{\rightarrow \infty} R_{2 n, \nu+1}(t) J_{\nu+1}(t) t^{-1} f(t) d t \\
& c_{n \nu}(N)=2(\nu+2 n+1) \int_{N}^{\rightarrow \infty} R_{2 n-1, \nu+2}(t) J_{\nu}(t) t^{-1} f(t) d t \\
& a_{n \nu}^{\prime}(a)=2(\nu+2 n+1) \int_{0}^{a} J_{\nu+2 n+1}(t) t^{-1} f(t) d t \\
& a_{n \nu}^{\prime \prime}(N)=b_{n \nu}(N)-c_{n \nu}(N) .
\end{aligned}
$$

It follows from the known inequality [2, p. 49]

$$
\left|J_{\mu}(t)\right| \leqq(t / 2)^{\mu} / \Gamma(\mu+1)
$$

valid when $\mu \geqq 0$, that $a_{n \nu}^{\prime}(a)$ exists when $f(t)$ is in $\mathcal{B}_{\nu}$ and

$$
\left|a_{n \nu}^{\prime}(a)\right| \leqq\left\{a^{2 n} / 2^{\nu+2 n} \Gamma(\nu+2 n+1)\right\} \int_{0}^{a} t^{\nu}|f(t)| d t .
$$

It follows from the definitions of the Lommel polynomials and Lemma 2.2 that the quantities $b_{n v}(N)$ and $c_{n v}(N)$ exist when $f(t)$ is in $\mathbb{B}_{\nu}$, and therefore $a_{n \nu}=a_{n \nu}^{\prime}(a)+b_{n \nu}(a)-c_{n \nu}(a)$ exists when $f(t)$ is in $\mathscr{B}_{\nu}$.

To prove the converse, suppose that $a_{n}$ exists $(n=0,1, \cdots)$. Since $a_{0}$, exists, $t^{-1} f(t) J_{\nu+1}(t)$ is in $L(0, a)$ for every positive $a$. Let $a_{1}$ be less than the smallest positive zero of $J_{v+1}(t)$. Then if $0 \leqq a \leqq a_{1}$, the function $t^{-1-\nu} J_{v+1}(t)$ has a positive lower bound $m_{\nu}$ on the interval $(0, a)$. The integrability of $t^{\nu} f(t)$ over the interval $(0, a)$ is then a consequence of the inequality 


$$
t^{\nu}|f(t)| \leqq m_{\nu}^{-1} t^{-1}|f(t)| J_{\nu+1}(t)
$$

valid when $0 \leqq t \leqq a$. If $a>a_{1}$, there is an integer $n$ such that $J_{\nu+2 n+1}(t)>0$ when $a_{1} \leqq t \leqq a$. We can in fact [2, p. 485] let $n$ be any integer for which $\nu+2 n+1>a$. The integrability of $t^{\nu} f(t)$ over the interval $\left(a_{1}, a\right)$ is now a consequence of the existence of $a_{n v}$. The limit (1.9) exists since $a_{0 v}$ exists. To see that the limit (1.10) exists we use the identity

$$
2(\nu+2) t^{-2} J_{\nu}(t)=-t^{-1} J_{\nu+3}(t)+\left[4 t^{-3}(\nu+1)(\nu+2)-t^{-1}\right] J_{\nu+1}(t) .
$$

Since $a_{0 v}$ and $a_{1 v}$ exist we can infer the existence of the limit (1.10) from Lemma 2.2.

Theorem 3.1 has thus been proved when $\nu>-1$. When $\nu \leqq-1$ every part of the direct proof is valid as stated above except the proof that $a_{n \nu}^{\prime}(a)$ exists. Since for any value of $\nu$

$$
J_{\nu+2 n+1}(t)=\sum_{m=0}^{\infty}(-1)^{m}(t / 2)^{\nu+2 n+1+2 m} / m ! \Gamma(m+\nu+2 n+2),
$$

the series converging uniformly when $0 \leqq t \leqq a$, and since

$$
t^{\nu+2 n+2 m}|f(t)| \leqq a^{2 n+2 m} t^{\nu}|f(t)|
$$

when $0 \leqq t \leqq a$, we can still conclude that $a_{n \nu}^{\prime}(a)$ exists, even though the inequality (3.2) may fail to hold. In the converse part of the theorem our proof that the limit (1.9) exists fails when $\nu=-1$ and that the limit (1.10) exists fails when $\nu=-2$. When $\nu=-1$ we use the identity

$$
3 t^{-1} J_{0}(t)=\left(24 t^{-3}-4 t^{-1}\right) J_{2}(t)-t^{-1} J_{4}(t) .
$$

Since $a_{1}$ and $a_{2 v}$ exist we can infer the existence of the limit (1.9) from Lemma 2.2. When $\nu=-2$ we use the identity

$$
8 t^{-2} J_{-2}(t)=-t^{-1} J_{5}(t)+\left(48 t^{-3}-t^{-1}\right) J_{3}(t) .
$$

Since $a_{2 v}$ and $a_{3}$, exist we can infer the existence of the limit (1.10) from Lemma 2.2.

In the rest of this section we shall derive some inequalities satisfied by $b_{n \nu}$ and $c_{n}$ when $\nu>-1$ which will be helpful in proving the main theorems. If we substitute the definition of $R_{2 n, v+1}(t)$ into the equation defining $b_{n v}(N)$ and use the second mean value theorem we find that

$$
b_{n \nu}(N)=2(\nu+2 n+1) \sum_{k=0}^{n} U_{k n}(N) \int_{N}^{\xi_{k}} t^{-1} f(t) J_{\nu+1}(t) d t
$$

in which $N<\xi_{k}$ and

$$
U_{k n}(N)=(-1)^{k}(N / 2)^{2 k-2 n} \Gamma(\nu+2 n-k+1)(2 n-k) ! / k !(2 n-2 k) ! \Gamma(\nu+1+k) .
$$


Similarly, we find that

$$
c_{n \nu}(N)=2(\nu+2 n+1) \sum_{k=0}^{n-1} V_{k n}(N) \int_{N}^{\eta_{k}} t^{-2} f(t) J_{\nu}(t) d t,
$$

in which $N<\eta_{k}$ and

$$
\begin{aligned}
V_{k n}(N)= & (-1)^{k} N(N / 2)^{2 k-2 n+1} \\
& \cdot \Gamma(\nu+1+2 n-k)(2 n-1-k) ! / k !(2 n-1-2 k) ! \Gamma(\nu+2+k) .
\end{aligned}
$$

Let us define

$$
M_{\nu}(N)=\max _{\xi \geqq N}\left|\int_{N}^{\xi} t^{-2} f(t) J_{\nu}(t) d t\right|+\max _{\xi \geqq N}\left|\int_{N}^{\xi} t^{-1} f(t) J_{\nu+1}(t) d t\right| .
$$

Then if $N \geqq 2 x$, it follows from the inequalities

$$
\begin{aligned}
& \sum_{k=0}^{n}\left|U_{k n}(N)\right| \leqq \sum_{k=0}^{n}\left|U_{k n}(2 x)\right|=(-1)^{n} R_{2 n, \nu+1}(2 i x) \\
& \sum_{k=0}^{n-1}\left|V_{k n}(N)\right| \leqq \sum_{k=0}^{n-1}\left|V_{k n}(2 x)\right|=2 i x(-1)^{n-1} R_{2 n-1, \nu+2}(2 i x)
\end{aligned}
$$

that

$$
\begin{aligned}
\left|b_{n \nu}(N)\right| \leqq 2(\nu+2 n+1) M_{\nu}(N)(-1)^{n} R_{2 n, \nu+1}(2 i x), \\
\left|c_{n \nu}(N)\right| \leqq 4(\nu+2 n+1) i x M_{\nu}(N)(-1)^{n-1} R_{2 n-1, \nu+2}(2 i x) .
\end{aligned}
$$

4. Proof of Theorem 1.1 with $\mathcal{A}_{\nu}$ replaced by $\mathcal{B}_{\nu}$. The following lemma has been proved earlier by the author [3, Lemma 5.1].

LemMA 4.1. If the series (1.1) converges when $x=\beta$, then the series (1.5) defines an analytic function $x^{-\nu} s_{\nu}(x)$ when $|x|<|\beta|$.

To prove the generalization of Theorem 1.1 it is therefore sufficient to show that the series (1.1) converges for each positive value of $x$ when $f(t)$ is in $\mathcal{B}_{\nu}$. Suppose temporarily that $\nu>-1$. It follows from the inequalities (3.1) and (3.2) that the series

$$
\sum_{n=0}^{\infty} a_{n v}^{\prime}(a) J_{\nu+2 n+1}(x)
$$

is dominated by the series

$$
\left(\frac{x}{2}\right)^{\nu+1}\left\{\sum_{n=0}^{\infty}\left(\frac{a x}{4}\right)^{2 n} / \Gamma(\nu+2 n+1) \Gamma(\nu+2 n+2)\right\} \int_{0}^{a} t^{\nu}|f(t)| d t
$$

and is therefore convergent when $a=2 x$. Moreover, since the inequality (3.3) holds, the series 


$$
\sum_{n=0}^{\infty} b_{r \nu}(N) J_{\nu+2 n+1}(x)
$$

is dominated by the series

$$
2 M_{\nu}(N) \sum_{n=0}^{\infty}(-1)^{n} R_{2 n, \nu+1}(2 i x)\left(\frac{x}{2}\right)^{\nu+2 n+1} / \Gamma(\nu+2 n+1)
$$

when $N \geqq 2 x$. It is known $[2$, p. 302] that

$$
\lim _{m=\infty}(i x)^{\mu+m} R_{m, \mu+1}(2 i x) / \Gamma(\mu+m+1)=J_{\mu}(2 i x)=i^{\mu} I_{\mu}(2 x) .
$$

Since $I_{\mu}(2 x)>0$ when $x>0$, there exists a function $\psi_{\mu}(x)$ such that

$$
\left|i^{m} x^{\mu+m} R_{m, \mu+1}(2 i x) / \Gamma(\mu+m+1) I_{\mu}(2 x)\right| \leqq \psi_{\mu}(x) .
$$

The series (4.3) is consequently dominated by the series

$$
2^{-\nu} M_{\nu}(N) I_{\nu}(2 x) \psi_{\nu}(x) x \sum_{n=0}^{\infty} 4^{-n},
$$

and is therefore convergent when $N=2 x$. Similarly, since the inequality (3.4) holds, the series

$$
\sum_{n=0}^{\infty} c_{n \nu}(N) J_{\nu+2 n+1}(x)
$$

is dominated when $N \geqq 2 x$ by the series

$$
2^{1-\nu} M_{\nu}(N) I_{\nu+1}(2 x) \psi_{\nu+1}(x) x^{2} \sum_{n=0}^{\infty} 4^{-n},
$$

and is therefore convergent when $N=2 x$. Since

$$
a_{n v}=a_{n v}^{\prime}(2 x)+b_{n \nu}(2 x)-c_{n \nu}(2 x),
$$

it follows that the series (1.1) converges for all positive $x$. This completes the proof of Theorem 1.1 with $\mathcal{A}_{\nu}$ replaced by $B_{\nu}$ when $\nu>-1$.

If $\nu \leqq-1$ we use the identity

$$
s_{\nu}(x)=\sum_{n=0}^{q-1} 2(\nu+2 n+1) J_{\nu+2 n+1}(x) \int_{0}^{\rightarrow \infty} t^{-1} f(t) J_{\nu+2 n+1}(t) d t+s_{\nu+2 q}(x),
$$

which is valid for any positive integer $q$. If $q=1+[(-\nu-1) / 2]$, then $q$ is positive and $\nu+2 q>-1$. Since $A_{\nu}^{*}$ is obviously a subset of $A_{\nu+2 q}^{*}$, it is clear that $B_{\nu}$ is a subset of $B_{v+2 q}$. It then follows from our previous analysis that $x^{-v-2 q} s_{v+2 q}$, and hence also $x^{-\nu} s_{\nu+2 q}$, is an entire function. Since the finite series in equation (4.8) obviously becomes an entire function after multiplication by 
$x^{-\nu}$, we conclude that $x^{-\nu} s_{\nu}(x)$ is an entire function for any value of $\nu$. These remarks complete the proof of Theorem 1.1 with $\mathcal{A}_{\nu}$ replaced by $\mathcal{B}_{\nu}$.

The analysis used to prove Theorem 1.1 may be extended slightly and used to prove the following lemma which asserts the possibility of interchanging the order of integration and summation in the definition of $s_{\nu}(x)$.

Lemma 4.2. If $f(t)$ is in $\mathrm{B}_{\boldsymbol{v}}$ and $x>0$, then

$$
s_{\nu}(x)=\int_{0}^{\rightarrow \infty} t^{-1} f(t) \phi_{\nu}(x, t) d t,
$$

in which

$$
\phi_{\nu}(x, t)=\sum_{n=0}^{\infty} 2(\nu+2 n+1) J_{\nu+2 n+1}(x) J_{\nu+2 n+1}(t) .
$$

Since the series $t^{-1-v} \phi_{\nu}(x, t)$ converges uniformly in $t$ on any finite interval, and since $t^{\nu} f(t)$ is integrable over any finite interval, it is sufficient to show that

(4.9) $\lim _{N=\infty} \sum_{n=0}^{\infty}\left|b_{n v}(N) J_{\nu+2 n+1}(x)\right|=0, \quad \lim _{N=\infty} \sum_{n=0}^{\infty}\left|c_{n \nu}(N) J_{\nu+2 n+1}(x)\right|=0$.

Since $\lim _{N=\infty} M_{\nu}(N)=0$ when $f(t)$ is in $\mathcal{B}_{\nu}$, the truth of the relations (4.9) when $\nu>-1$ is an immediate consequence of the fact that the series (4.3) and (4.6) are dominated respectively by the series (4.5) and (4.7). When $\nu \leqq-1$ we merely apply the idea behind equation (4.8) to see that the relations (4.9) still hold.

For future reference we note as a consequence of the fact that the series (4.1) is dominated by the series (4.2) that

$$
\lim _{a=0} \sum_{n=0}^{\infty}\left|a_{n \nu}^{\prime}(a) J_{\nu+2 n+1}(x)\right|=0
$$

when $\nu>-1$. It is easy to see that equation (4.10) also holds when $\nu \leqq-1$.

5. Proof of Theorem 1.2 and Theorem 1.3 with $\mathcal{A}_{\nu}$ replaced by $B_{\nu}$. The proof of Theorem 1.2 is formally the same whether $f(t)$ belongs to $A_{\nu}$ or to $\mathcal{B}_{v}$. The only part of this formal manipulation $[2$, p. 534], $[3$, p. 375] that needs examination is the proof that the integral

$$
\int_{0}^{\rightarrow \infty} L_{\nu}(t, v) f(t) d t
$$

converges uniformly in $v$ on the interval $(0, x)$ when

$$
L_{\nu}(t, v)=(t-v)^{-1} J_{1}(t-v)+(-1)^{v}(t+v)^{-1} J_{1}(t+v)
$$

and $f(t)$ is in $\mathcal{B}_{\boldsymbol{v}}$. We use the identities [2, pp. 145, 143] 


$$
\begin{aligned}
& J_{1}(t-v)=\sum_{m=-\infty}^{\infty} J_{m+1}(t) J_{m}(v) \\
& J_{1}(t+v)=\sum_{m=-\infty}^{\infty} J_{1-m}(t) J_{m}(v)=\sum_{m=-\infty}^{\infty}(-1)^{m} J_{m+1}(t) J_{m}(v),
\end{aligned}
$$

in which the right-hand sides are uniformly convergent in $t$ and $v$ on the set $\Delta$ defined by the inequalities $N^{\prime} \geqq t \geqq 2 x \geqq 2 v$ for any fixed $x$ and $N^{\prime}$. Expanding the terms $(t \pm v)^{-1}$ into power series in $v$ we thus find that

$$
L_{v}(t, v)=\sum_{m=-\infty}^{\infty} \sum_{p=0}^{\infty} J_{m+1}(t) J_{m}(v) v^{p}\left[1+(-1)^{m+p+\nu}\right] / t^{p+1},
$$

the double series being uniformly convergent on $\Delta$. If Neumann's factor $\epsilon_{m}=1+\delta_{m 0}$ be introduced [2, p. 22], we find that

$$
\begin{aligned}
L_{\nu}(t, v) & =\sum_{m=0}^{\infty} \sum_{p=0}^{\infty} \epsilon_{m}\left[J_{m+1}(t)-J_{m-1}(t)\right] J_{m}(v) v^{p}\left[1+(-1)^{m+p+\nu}\right] / 2 t^{p+1} \\
& =\sum_{m=0}^{\infty} \sum_{p=\nu}^{\infty} \epsilon_{m}\left[J_{m+1}(t)-J_{m-1}(t)\right] J_{m}(v) v^{p-\nu}\left[1+(-1)^{m+p}\right] / 2 t^{p+1-\nu}
\end{aligned}
$$

Remembering that $\nu$ is either 0 or -1 , we split the sum over $m$ into a sum over even $m$ and a sum over odd $m$. In this way we obtain

$$
L_{v}(t, v)=L_{v 1}(t, v)-L_{v 2}(t, v)+L_{v 3}(t, v)-L_{v 4}(t, v),
$$

in which for example

$$
L_{\nu 1}(t, v)=\sum_{m=0}^{\infty} \sum_{p=0}^{\infty} \epsilon_{2 m} J_{2 m+1}(t) J_{2 m}(v) v^{2 p-v} / t^{2 p+1-\nu} .
$$

If we use identities like

$$
J_{2 m+1}(t)=J_{\nu+1}(t) R_{2 m-\nu, \nu+1}(t)-J_{\nu}(t) R_{2 m-\nu-1, \nu+2}(t),
$$

it is seen that $L_{v j}(t, v)=L_{\nu j}^{\prime}(t, v)-L_{v j}^{\prime \prime}(t, v)$ where for example

$$
L_{\nu 1}^{\prime}(t, v)=\sum_{m=0}^{\infty} \sum_{p=0}^{\infty} \epsilon_{2 m} J_{\nu+1}(t) R_{2 m-\nu, \nu+1}(t) J_{2 m}(v) v^{2 p-\nu} / t^{2 p+1-\nu} .
$$

The existence and uniform convergence of the integral (5.1) will then be a consequence of the existence and uniform convergence of eight integrals like (5.1) with $L_{v}$ replaced by $L_{v j}^{\prime}$ and $L_{v j}^{\prime \prime}$. We shall now prove that

$$
\lim _{N, N^{\prime}=\infty} \int_{N}^{N^{\prime}} L_{\nu 1}^{\prime}(t, v) f(t) d t=\lim A\left(N, N^{\prime}\right)=0
$$

uniformly in $v$ on the interval $(0, x)$. The other seven integrals may be 
handled by similar methods. If we use the definition of $R_{2 m-v, v+1}$ and the second theorem of the mean as in $\$ 3$, we find that

$$
\begin{aligned}
\left|\int_{N}^{N^{\prime}} J_{\nu+1}(t) R_{2 m-\nu, \nu+1}(t) f(t) t^{\nu-1-2 p} d t\right| & \leqq N^{\nu-2 p} M_{\nu}(N) i^{2 m-\nu} R_{2 m-\nu, \nu+1}(i N) \\
& \leqq(2 v)^{\nu-2 p} M_{\nu}(N) i^{2 m-\nu} R_{2 m-\nu, \nu+1}(2 i v)
\end{aligned}
$$

whenever $N^{\prime} \geqq N \geqq 2 v$. If we now use the inequalities (4.4) and (3.1) we see that

$$
\left|A\left(N, N^{\prime}\right)\right| \leqq M_{\nu}(N) I_{\nu}(2 v) \psi_{\nu}(v) \sum_{m=0}^{\infty} \sum_{p=0}^{\infty} \epsilon_{2 m}\left(\frac{1}{2}\right)^{2 m+2 p-\nu} .
$$

Since $\psi_{\nu}(v) I_{\nu}(2 v)$ is bounded when $0 \leqq v \leqq x$ and since $M_{\nu}(N)$ approaches 0 as $N$ approaches $\infty$ when $f(t)$ is in $\mathcal{B}_{\nu}$, it follows that $A\left(N, N^{\prime}\right)$ approaches 0 as $N$ and $N^{\prime}$ approach $\infty$, uniformly in $v$.

The proof of Theorem 1.3 with $A_{\nu}$ replaced by $\mathcal{B}_{\nu}$ is also formally the same. Let $f(N, a, t)$ be $f(t)$ if $a \leqq t \leqq N$ and zero otherwise. Let $s_{\nu}(N, a, x)$ be the Neumann series expansion for $f(N, a, t)$. Just as in [3, p. 380] we find that

$$
s_{\nu}(N, a, x)=\{f(x+)+f(x-)\} / 2-x \int_{1}^{\rightarrow \infty} r J_{\nu}(x r) d r \int_{a}^{N} f(t) J_{\nu}(t r) d t .
$$

Since it follows from equations (4.9) and (4.10) that

$$
\lim _{a=0, N=\infty} s_{\nu}(N, a, x)=s_{\nu}(x)
$$

when $f(t)$ is in $B_{\nu}$, we conclude that Theorem 1.3 remains true when $A_{\nu}$ is replaced by $B_{\nu}$.

6. The proof that $A_{\nu}$ is properly contained in $A_{\nu}^{*}$. It is obvious that $A_{\nu}$ is a subset of $A_{\nu}^{*}$. If $A_{\nu}$ were not a proper subset of $A_{\nu}^{*}$, the material of this paper would be merely an alternative and simpler derivation of the results obtained in [3]. We shall now show that the function $f(t)$ $=t^{3 / 2} e^{t} \cos \left(e^{2 t}\right) g(t)$ lies in $A_{\nu}^{*}$ but not in $A_{\nu}$ provided that $g(t)=t^{-\nu}$ when $0 \leqq t \leqq 1$ and $g(t)=1$ when $t \geqq 1$. It is obvious that $t^{\nu} f(t)$ is in $L(0, a)$ for any positive $a$. The existence of the integrals

$$
\int_{0}^{\rightarrow \infty} e^{t} \cos \left(e^{2 t} \pm t\right) d t, \quad \int_{0}^{\rightarrow \infty} e^{t} \sin \left(e^{2 t} \pm t\right) d t
$$

can be proved with the help of a lemma of Titchmarsh [1, p. 22]. The integral (1.3) and the integral

$$
\int_{a}^{\rightarrow \infty} e^{t} \cos \left(e^{2 t}\right) \sin \left(t-\frac{\nu \pi}{2}+\frac{\pi}{4}\right) d t
$$


whose integrands are linear combinations of the integrands in (6.1), therefore exist. It follows from Lemma 2.2 that the integral (1.4) also exists. Hence $f(t)$ is in $A_{*}^{*}$.

To see that $f(t)$ is not in $\mathcal{A}_{\text {, }}$ it is sufficient to show that

$$
K_{p}=\int_{1 / 2}^{\infty} 2^{1-p} t^{-p} e^{t}\left|\cos e^{2 t}\right| d t=+\infty
$$

whenever $p \geqq 0$. The substitution $u=e^{2 t}$ shows that

$$
K_{p}=\int_{e}^{\infty}(\log u)^{-p} u^{-1 / 2}|\cos u| d u \equiv \int_{e}^{\infty} \phi(u) d u .
$$

Using the first theorem of the mean, we conclude that

$$
\int_{(4 n+3) \pi / 2}^{(4 n+5) \pi / 2} \phi(u) d u=2 / u_{n}^{1 / 2}\left(\log u_{n}\right)^{p}
$$

where $(4 n+3) \pi / 2<u_{n}<(4 n+5) \pi / 2$. If $n \geqq 16, n \geqq \exp \left[(\log 7) /\left(2^{1 / p}-1\right)\right]$, then $u_{n} \leqq 7 n, \log u_{n} \leqq 2^{1 / p} \log n$, and so

$$
\int_{(4 n+3) \pi / 2}^{(4 n+5) \pi / 2} \phi(u) d u \geqq 1 /(7 n)^{1 / 2}(\log n)^{p} .
$$

Similarly, we have that

$$
\int_{(4 n+5) \pi / 2}^{(4 n+7) \pi / 2} \phi(u) d u=2 / v_{n}^{1 / 2}\left(\log v_{n}\right)^{p} \geqq 1 /(7 n)^{1 / 2}(\log n)^{p},
$$

where $(4 n+5) \pi / 2 \leqq v_{n} \leqq(4 n+7) \pi / 2$ and $n$ satisfies the inequalities written above. The divergence of the integral $K_{p}$ is now a consequence of the divergence of the series

$$
\sum_{n=2}^{\infty} 1 / n^{1 / 2}(\log n)^{p}
$$

for any positive integer $p$.

\section{Bibliography}

1. E. C. Titchmarsh, Theory of the Fourier integral, Oxford, 1939.

2. G. N. Watson, $A$ treatise on the theory of Bessel functions, Cambridge, 1944.

3. J. E. Wilkins, Jr., Neumann series of Bessel functions, Trans. Amer. Math. Soc. vol. 64 (1948) pp. 359-385.

\section{American Optical Company,} Buffalo, N. Y. 